\title{
Free fall and cellular automata
}

\author{
Pablo Arrighi \\ Aix-Marseille Univ., LIF, F-13288 Marseille Cedex 9, France. \\ pablo.arrighi@univ-amu.fr \\ Gilles Dowek \\ Inria, LSV, ENS-Cachan, 61 Avenue du Président Wilson, 94230 Cachan, France. \\ gilles.dowek@inria.fr
}

\begin{abstract}
Three reasonable hypotheses lead to the thesis that physical phenomena can be described and simulated with cellular automata. In this work, we attempt to describe the motion of a particle upon which a constant force is applied, with a cellular automaton, in Newtonian physics, in Special Relativity, and in General Relativity. The results are very different for these three theories.
\end{abstract}

\section{Introduction}

Three reasonable hypotheses-homogeneity in time and space, bounded velocity of propagation of information, and bounded density of information-lead to the thesis that physical phenomena can be described and simulated with cellular automata. This implication has in fact been formalized into a theorem both in the classical [6] and the quantum case [1], albeit in flat space.

Further evaluating this thesis leads to the project of selecting specific physical phenomena and attempting to describe them with cellular automata. In this work, we consider a particle upon which a constant force is applied - as induced by the first order approximation of a gravitational field. We do so in three different settings: Newtonian physics, Special Relativity, and General Relativity. We seek to capture each of these motions as a Cellular Automaton. The results are very different for these three theories.

\section{Motion in cellular automata}

Recall that the configurations of a 1D cellular automaton are functions from $\mathbb{Z}$ to a finite set of states $\Sigma$, which includes a distinguished quiescent state $q$. The evolution of the cellular automaton is a function $F$ mapping configurations to configurations. It has to be causal and homogeneous, that is there must exist a radius $r$ and a local function $f$ such that for all $i,(F(\delta))_{i}=f\left(\delta_{i-r}, \ldots, \delta_{i-1}, \delta_{i}, \delta_{i+1}, \ldots, \delta_{i+r}\right)$.

Consider a temporal step $\varepsilon$, a spatial step $\Delta$, and a discrete motion $\tilde{y}$, that is a function from $\varepsilon \mathbb{N}$ to $\Delta \mathbb{Z}$. A configuration $\delta$ is said to represent a particle at position $k \Delta$ if $\delta_{k} \neq q$ and for all $i \neq k, \delta_{i}=q$. A transition function $F$ represents a discrete motion $\tilde{y}$ if there exists an initial configuration $\delta$ such that for all $k, F^{k}(\delta)$ represents a particle at $\tilde{y}(k \varepsilon)$. A standard reference on cellular automata for constructing signals approximating different functions is [7].

In each of the following three sections we proceed by first calculating the continuous motion $y(t)$, that is the position as a function of time. We then construct the cellular automata for $\tilde{y}$, if it exists. The differences between the three cases are highlighted.

C.A. Muñoz and J. A. Pérez (Eds.) :

Developments in Computational Models

EPTCS 204, 2016, pp. 1-10 doi 10.4204/EPTCS.204.1 (c) P. Arrighi \& G. Dowek

This work is licensed under the Creative Commons Attribution License. 


\section{Free fall in Newtonian physics}

We begin with the standard case of Newtonian physics. The choice of units and notations will carry through in the next sections. In Newtonian physics, the gravitational force applied by a body of mass $M$ upon a particle of mass $M^{\prime}$ at a distance $d$ is

$$
F=\mathscr{G} \frac{M M^{\prime}}{d^{2}}
$$

Multiplying both sides by $\mathscr{G} / c^{4}$, where $c$ is the speed of light, introducing notations $m=\left(\mathscr{G} / c^{2}\right) M$ which is the mass of the body expressed in meters, $m^{\prime}=\left(\mathscr{G} / c^{2}\right) M^{\prime}$ which is the mass of the particle expressed in meters, and $f=\left(\mathscr{G} / c^{4}\right) F$ which is the force expressed as a scalar without dimension, we get

$$
f=m^{\prime} \frac{m}{d^{2}}
$$

Consider a particle whose initial distance to the body is $R$ and initial velocity is zero and let $y$ be such that $d=R-y$, we have

$$
f=m^{\prime} \frac{m}{(R-y)^{2}}
$$

To define free fall, we approximate this force by

$$
f=m^{\prime} \frac{m}{R^{2}}
$$

that is, introducing the notation $g=m / R^{2}$

$$
f=m^{\prime} g
$$

For example, the mass of the Earth is $M=5.9710^{24} \mathrm{~kg}$, so $m=\left(\mathscr{G} / c^{2}\right) M=4.4210^{-3} \mathrm{~m}$. The radius of the Earth is $R=6.3710^{6} \mathrm{~m}$, so $g=m / R^{2}=1.0910^{-16} \mathrm{~m}^{-1}$. Note that $g c^{2}=9.81 \mathrm{~ms}^{-2}$ as expected.

When such a constant force is acting on a particle of mass $M^{\prime}$, its acceleration $A$ is given the equation

$$
M^{\prime} A=F
$$

Multiplying both sides with $\mathscr{G} / c^{4}$ and introducing the notation $a=A / c^{2}$, which is the acceleration of the particle expressed in $\mathrm{m}^{-1}$, we get

$$
m^{\prime} a=f=m^{\prime} g
$$

thus

$$
a=g
$$

from which we get

$$
v=g t
$$

where $v=V / c$ is the velocity expressed as a scalar with no dimension and $t=c T$ is time expressed in meters, and

$$
y=\frac{1}{2} g t^{2}
$$

Thus the spacetime trajectory of this particle is a parabola.

It is easy to prove that no cellular automaton can simulate such a motion: as the velocity of the particle increases linearly with time, the difference between $y$ at some time step and at the next time step increases linearly with time. Thus, the evolution is not local. Moreover, to be able to compute $y$ at the next time step from $y$ at some time step, we need to know the velocity of the particle and it is then natural to express this velocity as part of the state of the cell. But then, as velocity is not bounded, the state space cannot be kept finite, even if velocity is defined with a finite precision. 


\section{Constant force in Special Relativity}

In Special Relativity, neither of these problems occurs: velocity is bounded, hence the evolution is local. And if the velocity is known with a finite precision, a finite state space suffices. Still, another worry remains. If the velocity at some time step is computed from the velocity at the previous one, and both velocities are approximate, errors can accumulate. As we shall see, it is possible to circumvent this problem, and have a non divergent discretization of the trajectory of the particle.

In Special Relativity, the proper acceleration [8] of a particle is

$$
A=\frac{1}{{\sqrt{1-V^{2} / c^{2}}}^{3}} \frac{d V}{d T}
$$

dividing both sides by $c^{2}$, we get

$$
a=\frac{1}{\sqrt{1-v^{2}}} \frac{d v}{d t}
$$

We assume that the force is as in Newtonian physics: $m^{\prime} a=f=m^{\prime} g$, so $a=g$, that is

$$
\frac{1}{\sqrt{1-v^{2}}} \frac{d v}{d t}=g
$$

This assumption, however, is now better understood as "constant force" than "free fall". We get

$$
\frac{d v}{d t}=g{\sqrt{1-v^{2}}}^{3} .
$$

Solving this equation, we get

$$
v=\frac{g t}{\sqrt{1+(g t)^{2}}}
$$

as the reader may check by differentiating (5) and comparing the result with (4) with $v$ substituted by (5). Then

$$
y=\frac{1}{g}\left(\sqrt{1+(g t)^{2}}-1\right) .
$$

But, to prepare the case of General Relativity, we can also introduce a proper time $\tau$ such that

$$
\frac{d t}{d \tau}=\frac{1}{\sqrt{1-v^{2}}}
$$

Like $v=d y / d t$, we can introduce the velocity $w=d y / d \tau$ and we have

$$
w=\frac{d y}{d \tau}=\frac{d y}{d t} \frac{d t}{d \tau}=\frac{v}{\sqrt{1-v^{2}}}
$$

and then

$$
\frac{d w}{d \tau}=\frac{d w}{d v} \frac{d v}{d t} \frac{d t}{d \tau}=\frac{1}{{\sqrt{1-v^{2}}}^{3}} g{\sqrt{1-v^{2}}}^{3} \frac{1}{\sqrt{1-v^{2}}}=g \frac{1}{\sqrt{1-v^{2}}}
$$

From $w=v / \sqrt{1-v^{2}}$, we get $v=w / \sqrt{1+w^{2}}$, thus

$$
\frac{d w}{d \tau}=g \sqrt{1+w^{2}}
$$


which is the equation of motion in terms of proper time.

In the same way, we have

$$
\frac{d t}{d \tau}=\sqrt{1+w^{2}}
$$

which is the equation describing the relation between coordinate time $t$ and proper time $\tau$.

Solving Equation (6), we get

$$
w=\sinh (g \tau)
$$

and

$$
y=\frac{1}{g}(\cosh (g \tau)-1)
$$

Equation (7) then becomes

$$
\frac{d t}{d \tau}=\cosh (g \tau)
$$

and integrating it, we get

$$
t=\frac{1}{g} \sinh (g \tau)
$$

from which we get

$$
y=\frac{1}{g}\left(\sqrt{1+(g t)^{2}}-1\right)
$$

as expected.

Note that the velocity $w=\sinh (g \tau)$ goes to infinity when $\tau$ does. But the mapping from coordinate time to proper time $\tau=(1 / g) \operatorname{arsinh}(g t)$ slows down in such a way that the velocity $v=g t / \sqrt{1+(g t)^{2}}$ remains bounded by 1 . Hence the particle never goes faster than light.

The spacetime trajectory of the particle is a branch of the hyperbola of equation

$$
(g y+1)^{2}-(g t)^{2}=1
$$

Thus, in Special Relativity, the spacetime trajectory of a particle upon which a constant force is applied is not a branch of a parabola, but a branch of an hyperbola and the problem of modeling the motion of such a particle, with a cellular automaton, boils down to that of the approximability of a branch of hyperbola.

The branch of hyperbola

$$
y=\frac{1}{g}\left(\sqrt{1+(g t)^{2}}-1\right)
$$

has an asymptote

$$
y^{\prime}=t-\frac{1}{g}
$$

with whom the difference is

$$
y-y^{\prime}=\frac{1}{g}\left(\sqrt{1+(g t)^{2}}-g t\right)=\frac{1}{g\left(\sqrt{1+(g t)^{2}}+g t\right)}
$$

As expected, $y-y^{\prime}$ decreases and goes to 0 , when $t$ goes to infinity. Moreover, if working with a space accuracy $\Delta$, the hyperbola and its asymptote become indistinguishable at a time $\theta$ verifying

$$
\Delta=\frac{1}{g} \sqrt{1+(g \theta)^{2}}-g \theta
$$


that is at time

$$
\theta=\frac{1-(g \Delta)^{2}}{2 g^{2} \Delta}
$$

Consider an integer $N$ and let $\Delta=(1 / g) / N$. As $N$ can be taken as large as we wish, $\Delta$ can be taken as small as we wish. Consider the discretization of spacetime with a temporal and spatial step $\Delta$. Consider the function $\tilde{y}$ from $\Delta \mathbb{N}$ to $\Delta \mathbb{Z}$ mapping every $k \Delta$ smaller than $\theta$ to the rounding of $y(k \Delta)$ in $\Delta \mathbb{Z}$ and every $k \Delta$ larger than $\theta$ to $y^{\prime}(k \Delta)=k \Delta-(1 / g)=(k-N) \Delta$.

Let us construct a one-dimensional cellular automaton which represents the discrete motion $\tilde{y}$. Set the state space $\Sigma=\{q, 0, \ldots, L-1, \infty\}$, with $L=\ulcorner\theta / \Delta\urcorner$. Let us denote by $c(k, \sigma)$ the configuration such that all cells are in state $q$ except the cell $k$ which is in state $\sigma$. If $\sigma \in\{0, \ldots, L-1\}$, the cellular automaton maps $c(k, \sigma)$ to either $c(k, \sigma+1)$ or $c(k+1, \sigma+1)$-assuming $(L-1)+1=\infty$-depending on whether $\tilde{y}((k+1) \Delta)-\tilde{y}(k \Delta)$ is equal to zero or to $\Delta$, and $c(k, \infty)$ to $c(k+1, \infty)$.

Note that the internal state can be seen as a clock, the state $k$ corresponding to the time $k \Delta$. It can also be seen as a representation of the momentum, as the momentum $p=m^{\prime} v / \sqrt{1-v^{2}}=m^{\prime} w=m^{\prime} g t$ grows linearly with time, the state $k$ representing the momentum $\mathrm{km}^{\prime} \mathrm{g \Delta}$. The state $\infty$ corresponds to the case where momentum is large enough, so that its influence on velocity can be neglected, and the motion of the particle can be approximated by a uniform motion at the speed of light.

The number of states needed to simulate the spacetime trajectory is

$$
l=2+\frac{\theta}{\Delta}=\frac{1}{2 g^{2} \Delta^{2}}+\frac{3}{2}
$$

If we assume that the number of bits that can be encoded in a cell of length $\Delta$ is $\Delta / \rho$, for some distance $\rho$, then, to encode $\log _{2}\left(1 /\left(2 g^{2} \Delta^{2}\right)+3 / 2\right)$ bits, we need a cell of size $\Delta$ such that

$$
\log _{2}\left(\frac{1}{2 g^{2} \Delta^{2}}+\frac{3}{2}\right) \leq \Delta / \rho
$$

that is

$$
\Delta / \rho-\log _{2}\left(\frac{1}{2 g^{2} \Delta^{2}}+\frac{3}{2}\right) \geq 0
$$

The function $\Delta / \rho-\log _{2}\left(1 /\left(2 g^{2} \Delta^{2}\right)+3 / 2\right)$ is monotonic in $\Delta$, so this equation can be numerically solved.

For example, if $g=1.0910^{-16} \mathrm{~m}^{-1}$ and $\rho=1.610^{-35} \mathrm{~m}$, this equation boils down to

$$
\Delta \geq 5.1110^{-33} \mathrm{~m}=320 \rho
$$

Indeed, if we take $\Delta=320 \rho$, a cell can encode 320 bits and $l=1.5410^{96}=2^{320}$.

So, with an accuracy of the order of magnitude of $10^{-33} \mathrm{~m}$, constant force in Special Relativity does not require a particle to contain more than a few hundred bits.

\section{Free fall in General Relativity}

In General Relativity, the gravitational effect of a body of mass $M$ at a distance $d=R-y$ is described by the metric tensor

$$
\left(\begin{array}{cc}
g_{t t} & 0 \\
0 & -\frac{1}{g_{t t}}
\end{array}\right)
$$

where $g_{t t}=1-2 m /(R-y)$. 
The motion of a particle is described as a function mapping its proper time $\tau$ to a point in spacetime $\left(\begin{array}{l}t(\tau) \\ y(\tau)\end{array}\right)$. The equations of this motion are [5]:

$$
\begin{gathered}
\frac{d^{2} t}{d \tau^{2}}+2 \Gamma_{y t}^{t} \frac{d t}{d \tau} \frac{d y}{d \tau}=0 \\
\frac{d^{2} y}{d \tau^{2}}+\Gamma_{t t}^{y}\left(\frac{d t}{d \tau}\right)^{2}+\Gamma_{y y}^{y}\left(\frac{d y}{d \tau}\right)^{2}=0
\end{gathered}
$$

where

$$
\begin{gathered}
\Gamma_{t t}^{y}=\frac{1}{2} g_{t t} \frac{d g_{t t}}{d y} \\
\Gamma_{y y}^{y}=-\frac{1}{2} \frac{1}{g_{t t}} \frac{d g_{t t}}{d y} \\
\Gamma_{y t}^{t}=\Gamma_{t y}^{t}=\frac{1}{2} \frac{1}{g_{t t}} \frac{d g_{t t}}{d y}
\end{gathered}
$$

are the non-zero Christoffel symbols corresponding to this metric tensor, that is

$$
\begin{gathered}
\frac{d^{2} t}{d \tau^{2}}=-\frac{1}{g_{t t}} \frac{d g_{t t}}{d y} \frac{d t}{d \tau} \frac{d y}{d \tau} \\
\frac{d^{2} y}{d \tau^{2}}=-\frac{1}{2} \frac{d g_{t t}}{d y}\left(g_{t t}\left(\frac{d t}{d \tau}\right)^{2}-\frac{1}{g_{t t}}\left(\frac{d y}{d \tau}\right)^{2}\right)
\end{gathered}
$$

to which we can add a third equation expressing that $\tau$ is a proper time

$$
g_{t t}\left(\frac{d t}{d \tau}\right)^{2}-\frac{1}{g_{t t}}\left(\frac{d y}{d \tau}\right)^{2}=1
$$

Note that adding this third equation permits to drop the first, because differentiating the third equation and using the second to replace $d^{2} y / d \tau^{2}$ by $-(1 / 2)\left(d g_{t t} / d y\right)\left(g_{t t}(d t / d \tau)^{2}-\left(1 / g_{t t}\right)(d y / d \tau)^{2}\right)$ yields the first. Using this third equation, the second can also be simplified to

$$
\frac{d^{2} y}{d \tau^{2}}=-\frac{1}{2} \frac{d g_{t t}}{d y}
$$

Thus, introducing the velocity $w=d y / d \tau$, the equations of motion boil down to the two equations

$$
\begin{gathered}
\frac{d w}{d \tau}=-\frac{1}{2} \frac{d g_{t t}}{d y} \\
\frac{d t}{d \tau}=\frac{1}{g_{t t}} \sqrt{g_{t t}+w^{2}}
\end{gathered}
$$

which are, respectively, the equation of motion in terms of proper time and that describing the relation between coordinate time $t$ and proper time $\tau$.

"Constant force due to free fall" would make for a non-standard concept in General Relativity. On the one hand, a constant force of non-gravitational origin could indeed be applied in flat space and lead to the exact same computations as Special Relativity. On the other hand, free-falling could just mean following 
a geodesic trajectory in some more or less complicated metric-although not in a constant one. Indeed, making $g_{t t}$ constant as in the approximation of Equation (1) into (2) becomes an over-approximation, as the geodesics then become linear. So, we define this "first order approximated free fall" as the first non-trivial approximation of the metric tensor, that is we take a linear approximation of $g_{t t}$ as

$$
g_{t t}=1-\frac{2 m}{R}-\frac{2 m}{R^{2}} y=1-\frac{2 m}{R}-2 g y
$$

where $g=m / R^{2}$ is the acceleration of gravity, as before. Introducing $y_{1}=(1-(2 m / R)) / 2 g$ we get

$$
g_{t t}=2 g\left(y_{1}-y\right)
$$

In the same way, we approximate $d g_{t t} / d y=-2 m /(R-y)^{2}$ by $-2 m / R^{2}=-2 g$.

The equations of motion then become

$$
\begin{gathered}
\frac{d w}{d \tau}=g \\
\frac{d t}{d \tau}=\frac{1}{2 g\left(y_{1}-y\right)} \sqrt{2 g\left(y_{1}-y\right)+w^{2}}
\end{gathered}
$$

Note the differences and similarities with the cases of the previous settings. The equation describing the relation between coordinate time and proper time, that is Equation (10) or (8) does coincide with that of Special Relativity, that is Equation (7), in the flat spacetime case when $g_{t t}=1$. But the equation of motion, that is Equation (9), coincides not with Special Relativity, that is Equation (6), but with Newtonian physics, that is (3).

Integrating Equation (9), we get

$$
w=g \tau
$$

and

$$
y=\frac{1}{2} g \tau^{2}
$$

Equation (10) then becomes

$$
\begin{gathered}
\frac{d t}{d \tau}=\frac{1}{2 g\left(y_{1}-(1 / 2) g \tau^{2}\right)} \sqrt{2 g\left(y_{1}-(1 / 2) g \tau^{2}\right)+(g \tau)^{2}} \\
\frac{d t}{d \tau}=\sqrt{\frac{y_{1}}{2 g}} \frac{1}{y_{1}-(1 / 2) g \tau^{2}}
\end{gathered}
$$

Integrating it, we obtain

$$
\begin{gathered}
t=\frac{1}{g} \operatorname{artanh}\left(\tau \sqrt{\frac{g}{2 y_{1}}}\right) \\
\tau=\sqrt{\frac{2 y_{1}}{g}} \tanh (g t)
\end{gathered}
$$

and finally

$$
y=\frac{1}{2} g \tau^{2}=y_{1}(\tanh (g t))^{2}
$$


Note that the velocity

$$
v=\frac{d y}{d t}=2 g y_{1} \tanh (g t)\left(1-(\tanh (g t))^{2}\right)=\left(1-\frac{2 m}{R}\right) \tanh (g t)\left(1-(\tanh (g t))^{2}\right)
$$

is bounded by 1 , hence the particle never goes faster than light.

Like in Special Relativity, the velocity $w=g \tau$ goes to infinity when $\tau$ does and the mapping from coordinate time to proper time

$$
\tau=\sqrt{\frac{2 y_{1}}{g}} \tanh (g t)
$$

slows down in such a way that the velocity $v$ is bounded by 1 . Moreover, unlike in Special Relativity, when $t$ goes to infinity, $\tau$ has a finite limit $\sqrt{2 y_{1} / g}$. Thus, an infinite amount of coordinate time corresponds to a finite amount of proper time. As a consequence, with respect to coordinate time, after an acceleration phase, the particle decelerates and has a limit position $y_{1}$.

The distance to the limit at time $t$ is

$$
y_{1}-y=y_{1}\left(1-(\tanh (g t))^{2}\right)
$$

As expected, $y_{1}-y$ decreases and goes to 0 when $t$ goes to infinity. Moreover, if working with a space accuracy of $\Delta$, the position and its limit become indistinguishable at a time $\theta$ verifying

$$
\Delta=y_{1}\left(1-(\tanh (g \theta))^{2}\right)
$$

that is at time

$$
\theta=\frac{1}{g} \operatorname{artanh}\left(\sqrt{1-\frac{\Delta}{y_{1}}}\right)
$$

Consider a distance $\Delta$ that can be taken as small as we wish. Like in the case of Special Relativity, consider the discretization of spacetime with a temporal and spatial step $\Delta$ and the function $\tilde{y}$ from $\Delta \mathbb{N}$ to $\Delta \mathbb{Z}$ mapping every $k \Delta$ smaller than $\theta$ to the rounding of $y(k \Delta)$ in $\Delta \mathbb{Z}$ and every $k \Delta$ larger than $\theta$ to $y_{1}$.

Let us construct a one-dimensional cellular automaton which represents the discrete motion $\tilde{y}$. Set the state space $\Sigma=\{q, 0, \ldots, L-1, \infty\}$, with $L=\ulcorner\theta / \Delta\urcorner$. If $\sigma \in\{0, \ldots, L-1\}$, the cellular automaton maps $c(k, \sigma)$ to either $c(k, \sigma+1)$ or $c(k+1, \sigma+1)$-assuming $(L-1)+1=\infty$-depending on whether $\tilde{y}((k+1) \Delta)-\tilde{y}(k \Delta)$ is equal to zero or to $\Delta$, and $c(k, \infty)$ to itself.

The number of states needed to simulate the spacetime trajectory is

$$
l=2+\theta / \Delta=2+\frac{1}{g \Delta} \operatorname{artanh}\left(\sqrt{1-\frac{\Delta}{y_{1}}}\right)
$$

If we assume that the number of bits that can be encoded in a cell of length $\Delta$ is $\Delta / \rho$, for some distance $\rho$, then, to encode this amount of information, we need a cell of size $\Delta$ where

$$
\log _{2}\left(2+\frac{1}{g \Delta} \operatorname{artanh}\left(\sqrt{1-\frac{\Delta}{y_{1}}}\right)\right) \leq \frac{\Delta}{\rho}
$$

that is

$$
\frac{\Delta}{\rho}-\log _{2}\left(2+\frac{1}{g \Delta} \operatorname{artanh}\left(\sqrt{1-\frac{\Delta}{y_{1}}}\right)\right) \geq 0
$$


This function is monotonic in $\Delta$, so this equation can be numerically solved. For example, if $m=$ $4.4210^{-3} \mathrm{~m}, R=6.3710^{6} \mathrm{~m}$, and $\rho=1.610^{-35} \mathrm{~m}$, we get $g=1.0910^{-16} \mathrm{~m}^{-1}$ and $y_{1}=4.5710^{15} \mathrm{~m}$. This equation boils down to

$$
\Delta \geq 2.6910^{-33} \mathrm{~m}=168 \rho
$$

Indeed, if we take $\Delta=168 \rho$, a cell can encode 168 bits and $l=1.9210^{50}=2^{168}$.

So, with an accuracy of the order of magnitude of $10^{-33} \mathrm{~m}$, General Relativity also does not require a free falling particle to contain more than a few hundred bits.

\section{Conclusion}

Newtonian physics and Relativity completely differ with respect to the possibility modelling free fall within a cellular automaton. Such a simulation is not possible for Newtonian physics, while it is possible both in Special—constant force—and General Relativity_geodesics in a linearly approximated metric. The simulation can be very accurate with a reasonable number of internal states: a few hundred bits suffice to achieve an accuracy of the order of magnitude of $10^{-33} \mathrm{~m}$. So, as far as free fall is concerned, Relativity is completely consistent with the hypotheses of a bounded velocity of propagation of information and of a bounded density of information, unlike Newtonian physics.

In this work, we made explicit these accurate cellular automata, by exploiting the asymptotes to the trajectory, that exist both in Special and General Relativity. There was no need to use auxiliary signals as in [7]. We have proved the existence of such cellular automata, but made no attempt to design "natural" ones: the local rules use the solutions of the equations of motion in order to know whether the particle should move, or not. Moreover, there was clearly no attention paid to covariance. The design of more natural automata is of course of prime importance. In the case of General Relativity for instance, the metric at each point ought to be carried by the corresponding cell: we began to address this question both in the classical case [2], and, building upon [4], in the quantum case [3].

\section{Acknowledgements}

The authors thank David Janin for useful discussions on this paper, and Alejandro Péres for indications on General Relativity. This work has been funded by the ANR-12-BS02-007-01 TARMAC grant. Pablo Arrighi is also a member of IXXI, where this research was partially conducted.

\section{References}

[1] P. Arrighi \& G. Dowek (2012): The physical Church-Turing thesis and the principles of quantum theory. Int. J. Found. of Computer Science 23, doi:10.1142/S0129054112500153.

[2] P. Arrighi \& G. Dowek (2015): Discrete geodesics. In: Theory and Practice of Natural Computing, Lecture Notes in Computer Science 9477, Springer-Verlag, pp. 137-149, doi 10.1007/978-3-319-26841-5_11.

[3] P. Arrighi, S. Facchini \& M. Forets (2015): Quantum walks in curved spacetime. Pre-print arXiv:1505.07023.

[4] G. Di Molfetta, M. Brachet \& F. Debbasch (2013): Quantum walks as massless Dirac fermions in curved space-time. Physical Review A 88(4), p. 042301, doi 10.1103/PhysRevA.88.042301.

[5] R. d'Inverno (1899): Introducing Einstein's Relatvity. Oxford University Press, USA.

[6] R. Gandy (1980): Church's thesis and principles for mechanisms. In: The Kleene Symposium, North-Holland Publishing Company, Amsterdam, pp. 123-148, doi 10.1016/S0049-237X(08)71257-6 
[7] J. Mazoyer \& V. Terrier (1999): Signals in one-dimensional cellular automata. Theoretical Computer Science 217(1), pp. 53 - 80, doi:10.1016/S0304-3975(98)00150-9 Available at http://www.sciencedirect.com/science/article/pii/S0304397598001509

[8] E.F. Taylor \& J.A. Wheeler (1992): Spacetime physics. Macmillan. 